Article

\title{
Friedel-Crafts-Type Alkylation of Indoles in Water Using Amphiphilic Resin-Supported 1,10-Phenanthroline-Palladium Complex under Aerobic Conditions
}

\author{
Toshimasa Suzuka *, Yuto Ooshiro and Kazuhito Ogihara \\ Department of Chemistry, Biology and Marine Science, University of the Ryukyus, Nishihara, \\ Okinawa 903-0213, Japan; k188362@eve.u-ryukyu.ac.jp (Y.O.); kogihara@sci.u-ryukyu.ac.jp (K.O.) \\ * Correspondence: suzuka@sci.u-ryukyu.ac.jp; Tel.: +81-98-895-8531
}

Received: 20 January 2020; Accepted: 4 February 2020; Published: 5 February 2020

check for updates

\begin{abstract}
The palladium-catalyzed Friedel-Crafts-type alkylation of indoles in water has been achieved using amphiphilic polystyrene-poly(ethylene glycol) (PS-PEG) resin-supported phenanthroline-palladium complexes in water under aerobic conditions, affording the corresponding products with good-to-high yield. The polymeric catalyst was also found to promote the C3-alkylation reaction to give a thermodynamic alkylation product with high selectivity. The polymeric catalyst was recovered and reused several times without any loss of catalytic activity.
\end{abstract}

Keywords: aqueous media; polymer-support; Friedel-Crafts reaction

\section{Introduction}

The Friedel-Crafts reaction is one of the considerably important carbon-carbon bond forming reactions to employ Lewis acids as promoters since the pioneering study by Friedel and Crafts [1,2]. Recently, the original procedure (for which stoichiometric amounts of a Lewis acid were required) has been replaced by catalytic Friedel-Crafts-type reactions for the alkylation and acylation of aromatic and heteroarene compounds [3,4]. While widespread research has been devoted to the catalytic Friedel-Crafts reactions of allyl compounds with electron-rich aromatics, research on the catalytic Friedel-Crafts reactions has took place in organic solvent [5-15] or in water solvent [16,17] under homogeneous conditions. If the Friedel-Crafts reactions were performed in water with recyclable palladium catalysts, where neither aqueous-organic solvent wastes nor metal-contaminated wastes were yielded, this would go a long way to meeting green chemical requirements.

Nowadays, we have developed amphiphilic polystyrene-poly(ethylene glycol) (PS-PEG) resin-supported terpyridine-palladium complexes that promote varied catalytic transformations [18-28] smoothly in water [29-32] under heterogeneous conditions [33-36]. Herein, we aimed for the Friedel-Crafts reaction of $\pi$-allylic palladium intermediates with indoles in water under aerobic conditions in the presence of amphiphilic PS-PEG resin-bound pyridine skeleton ligand-palladium complexes 1a-c (Scheme 1). 


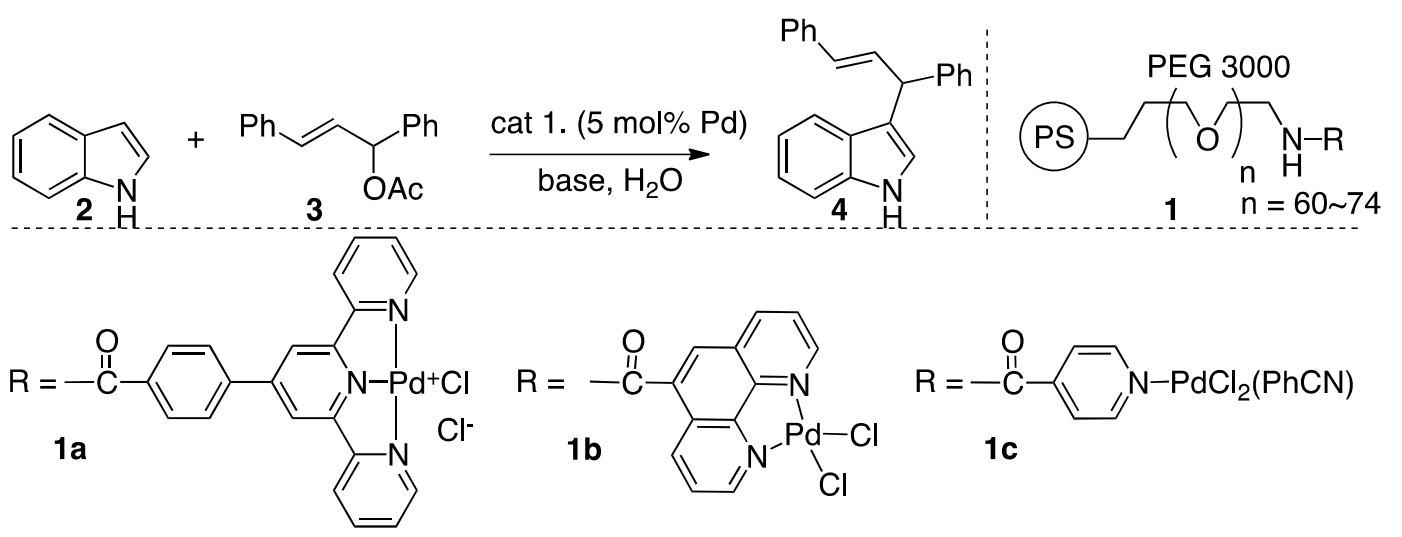

Scheme 1. Friedel-Crafts-type alkylation of indole in water using polymeric catalysts 1.

\section{Results}

\section{Alkylation Reaction}

First, the polymeric catalyst $\mathbf{1 b}$ was prepared from phenanthroline carboxylic acid, PS-PEG resin, and palladium in accordance with previously reported procedures [26], and we then examined different bases and catalysts in the alkylation reaction in water to distinguish which bases and catalysts were most suitable for use in the reaction (Table 1). Thus, the alkylation reaction of indole (2a) and 1,3-diphenyl-2-propenyl acetate (3a) was carried out in water with $\mathrm{Et}_{3} \mathrm{~N}$ (3.0 equivalent) in the presence of the polymeric catalyst $\mathbf{1 b}\left(5 \mathrm{~mol} . \%\right.$ to $\mathrm{Pd}$ ) at $40{ }^{\circ} \mathrm{C}$ for $24 \mathrm{~h}$. After completion of the reaction, the reaction mixture was filtered, and the recovered resin beads were rinsed with a small portion of water and extracted with EtOAc to give an $88 \%$ yield of 3-(1,3-diphenyl-2-propenyl)- $1 \mathrm{H}$-indole (4a) (entry 2, Table 1). The scope of suitable bases for the C3-alkylation of indole in water using catalyst $\mathbf{1} \mathbf{b}$ was examined. lithium carbonate, sodium carbonate, potassium carbonate, cesium carbonate, sodium hydrogen carbonate, and DBU gave $27 \%, 12 \%, 13 \%, 16 \%, 28 \%$, and $30 \%$ yields, respectively (entries 4-9, Table 1). Next, we tested several catalysts using $\mathrm{Et}_{3} \mathrm{~N}$ as a base for the C3-alkylation reaction, which produced $4 \mathbf{a}$ with $43 \%-88 \%$ yields (entries 1-3, Table 1 ). The most effective catalyst proved to be polymer-supported 1,10-phenanthroline-palladium complex $\mathbf{1 b}$, which gave $4 \mathbf{a}$ in an impressive $88 \%$ yield (entry 2, Table 1). In addition, these reaction conditions were applicable to scale up the reaction (entry 10, Table 1). Thus, the reaction was performed using 20 times the amount of indole of entry 2 to give the corresponding product in $94 \%$ yield.

With the optimal conditions in hand, we examined the polymer-supported 1,10-phenanthrolinepalladium catalyzed alkylation of several indoles, and the results are summarized in Table 2. The 2-methylindole gave a $78 \%$ yield of 3-allyl-2-methyl- $1 H$-indole $4 \mathbf{b}$ (entry 2, Table 2). Due to the low dissolubility of 2-phenylindole (2c), the reaction of the $2 \mathrm{c}$ with $3 \mathbf{a}$ afforded the 3-allyl-2-phenyl-1H-indole $4 \mathbf{c}$ in only a $27 \%$ yield (entry 3, Table 2). The 5-methylindole (2d) and 7-methylindole (2e) also underwent the alkylation to give $4 \mathbf{d}$ and $4 \mathbf{e}$ in $91 \%$ and $77 \%$ yields, respectively (entries 4 and 5, Table 2). The alkylation of indoles $\mathbf{2 f}$ and $\mathbf{2 g}$ having an electron withdrawing group at the 5-position afforded the 3-allyl-5-bromo- $1 \mathrm{H}$-indole $4 \mathrm{f}$ and 3-allyl-2-chloro- $1 \mathrm{H}$-indole $4 \mathrm{~g}$ in $74 \%$ and $64 \%$ yields, respectively (entries 6 and 7, Table 2). The 5-substituted indoles $\mathbf{2 h}$ and $2 \mathbf{i}$ having an electron donating group $\left(\mathrm{OCH}_{3}\right.$ or $\left.\mathrm{OBn}\right)$ furnished the 3-allyl-5-methoxy- $1 \mathrm{H}$-indole $4 \mathrm{~h}$ and 3-allyl-2-benzyloxy- $1 \mathrm{H}$-indole $4 \mathbf{i}$ in $52 \%$ and $33 \%$ yields, respectively (entries 8 and 9 , Table 2 ). The cyclic substrate was also examined, but the alkylation reaction did not proceed at all (entry 10). 
Table 1. Friedel-Crafts-type alkylation of indole in water using catalysts $\mathbf{1}$ and several bases ${ }^{\text {a }}$.
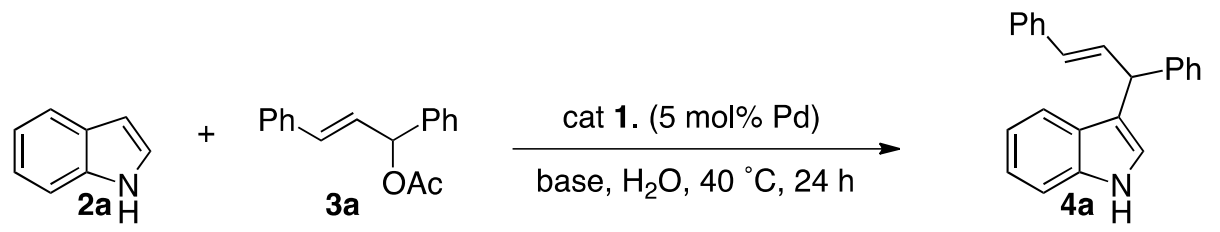

\begin{tabular}{cccc}
\hline Entry & Catalyst & Bases (3 equiv.) & Yield of 4a (\%) \\
\hline 1 & $\mathbf{1 a}$ & $\mathrm{Et}_{3} \mathrm{~N}$ & 43 \\
2 & $\mathbf{1 b}$ & $\mathrm{Et}_{3} \mathrm{~N}$ & 88 \\
3 & $\mathbf{1 c}$ & $\mathrm{Et}_{3} \mathrm{~N}$ & 53 \\
4 & $\mathbf{1 b}$ & $\mathrm{Li}_{2} \mathrm{CO}_{3}$ & 27 \\
5 & $\mathbf{1 b}$ & $\mathrm{Na}_{2} \mathrm{CO}_{3}$ & 12 \\
6 & $\mathbf{1 b}$ & $\mathrm{K}_{2} \mathrm{CO}_{3}$ & 13 \\
7 & $\mathbf{1 b}$ & $\mathrm{Cs}_{2} \mathrm{CO}_{3}$ & 16 \\
8 & $\mathbf{1 b}$ & $\mathrm{NaHCO}_{3}$ & 28 \\
9 & $\mathbf{1 b}$ & $\mathrm{DBU}$ & 30 \\
$10^{\mathrm{b}}$ & $\mathbf{1 b}$ & $\mathrm{Et}_{3} \mathrm{~N}$ & $94^{\mathrm{c}}$ \\
\hline
\end{tabular}

a: All reactions were performed in water at $40{ }^{\circ} \mathrm{C}$ for $24 \mathrm{~h}$. The ratio of $3(\mathrm{~mol}) /$ indoles $(\mathrm{mol}) / \mathrm{base}(\mathrm{mol}) / \mathrm{Pd}$ $(\mathrm{mol}) / \mathrm{H}_{2} \mathrm{O}(\mathrm{mL})=1.5 / 1 / 3 / 0.05 / 1.5$. Yields were determined by gas chromatography $(\mathrm{GC})$ based on $n$-dodecane as an internal standard. ${ }^{b}$ : This reaction was performed under scaled-up conditions $(3 \mathrm{mmol}) /$ indoles $(2 \mathrm{mmol}) / \mathrm{base}$

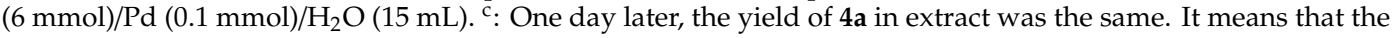
reaction did not proceed without the polymeric catalyst.

Table 2. Friedel-Crafts-type alkylation of indoles 2 with allyl esters 3 in $\mathrm{H}_{2} \mathrm{O}$.

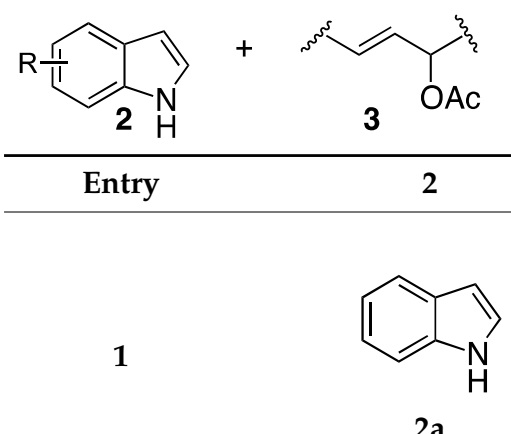

2a<smiles>Cc1cc2ccccc2[nH]1</smiles>

$2 b$<smiles>CC(C)(C)c1ccccc1</smiles>

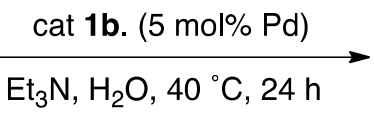

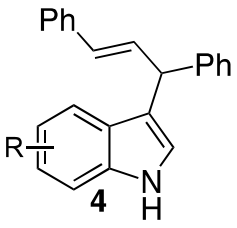

Yield (\%) ${ }^{a}$<smiles>C(=C/c1c[nH]c2ccccc12)\c1ccccc1</smiles>

88

78<smiles>Cc1[nH]c2ccccc2c1C(/C=C/c1ccccc1)c1ccccc1</smiles>

$4 b$<smiles></smiles>

27 
Table 2. Cont.

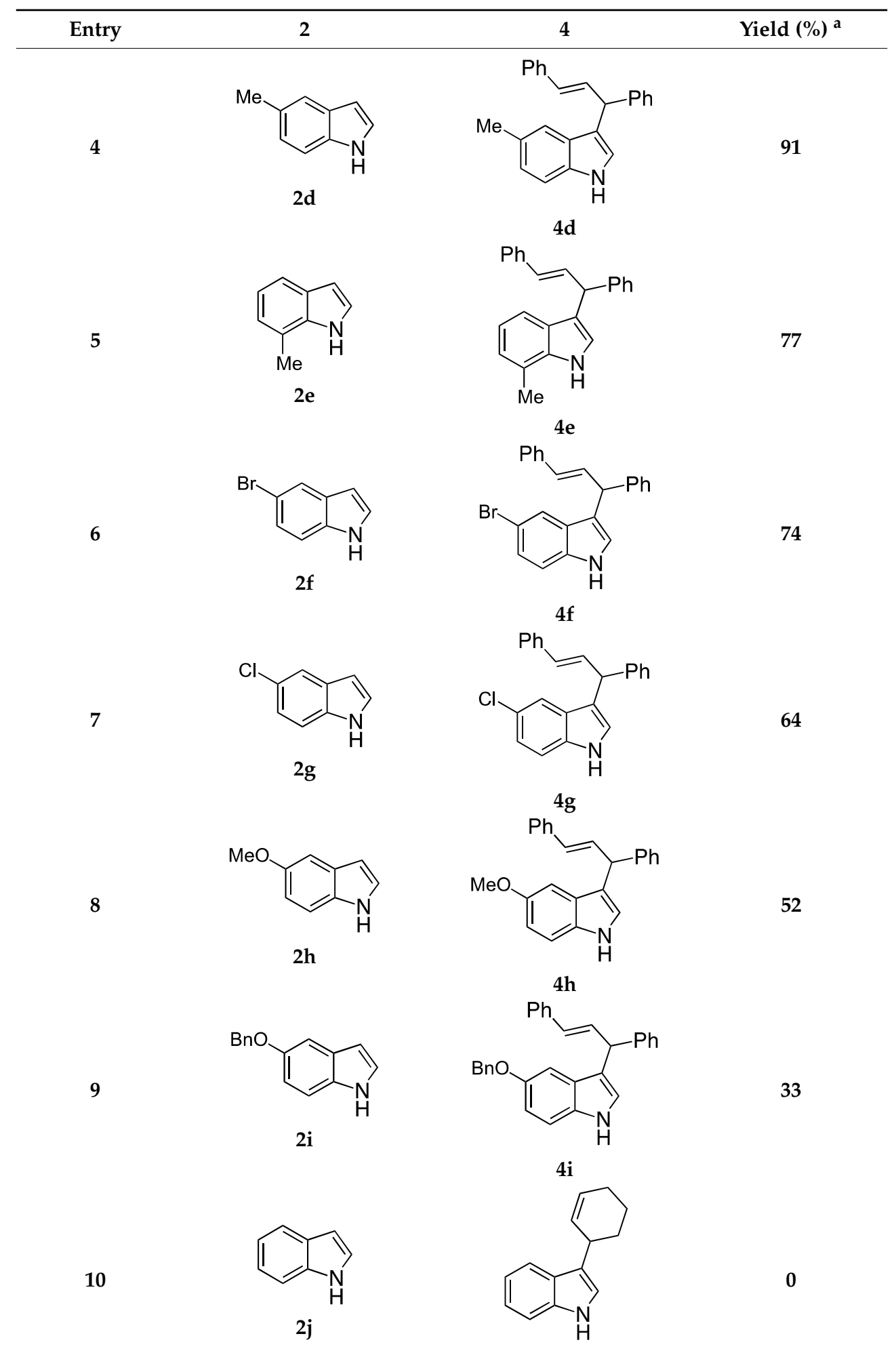

$4 \mathbf{j}$

a: All reactions were performed in water at $40^{\circ} \mathrm{C}$ for $24 \mathrm{~h}$. The ratio of $\mathbf{3}(\mathrm{mol}) /$ indoles $(\mathrm{mol}) /$ triethylamine $(\mathrm{mol}) / \mathrm{Pd}$ $(\mathrm{mol}) / \mathrm{H}_{2} \mathrm{O}(\mathrm{mL})=1.5 / 1 / 3 / 0.05 / 1.5$.

Recycling experiments were tested for alkylation of indole (2a) with the allyl ester 3a. After the first use of the polymeric palladium catalyst $1 \mathbf{b}$ (Table 1 , entry 1 ) to give an $88 \%$ yield of the C3-allylindole $4 \mathbf{a}$, the recovered catalyst beads were taken on for a 3rd reuse and exhibited stable 
catalytic activity (Scheme 2). After the recycling experiments, inductively coupled plasma-atomic emission spectrometry (ICP-AES) analysis showed that the concentration of Pd leached into the aqueous solution was $<0.3 \mathrm{ppm}$.

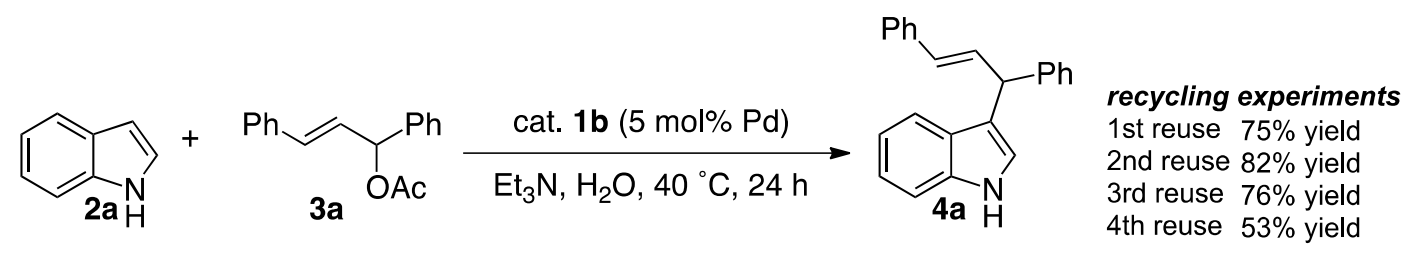

Scheme 2. Recycling experiments.

Bandini and co-workers reported that while low coordinating solvents would favor the generation of C3-alkylation product $4 a$, the use of highly coordinating solvents would drive the regiochemistry toward the formation of $\mathrm{N}$-alkylation product 5 (Scheme 3) [9]. It is noteworthy that the C3-allylindole $4 \mathbf{a}$ was obtained as a sole product using polymeric catalyst $\mathbf{1 b}$ with triethylamine in water. These results suggested that the Friedel-Crafts-type reaction of indole in water with polymeric catalyst $\mathbf{1 b}$ proceeded in the polystyrene moiety of the polystyrene-poly(ethylene glycol) matrix to give thermodynamic alkylation product $4 \mathrm{a}$ in $88 \%$ yield.

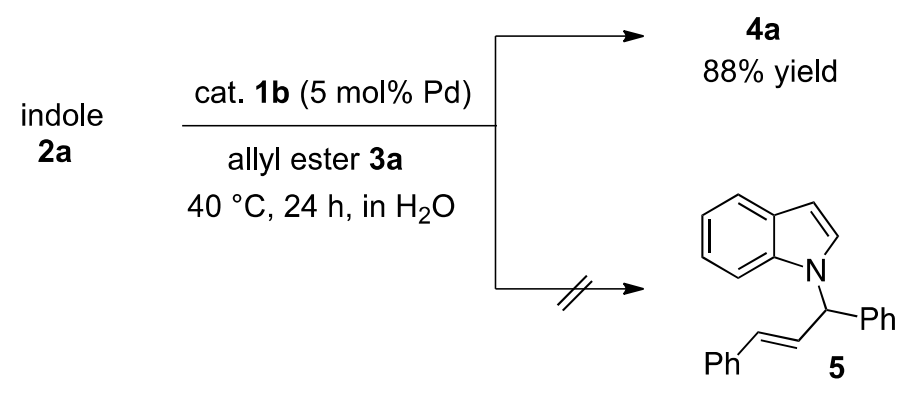

Scheme 3. Regioselective alkylation of indole.

\section{Materials and Methods}

\subsection{General Methods}

All manipulations were conducted under aerobic conditions. Water was deionized with a Millipore Milli-Q Gradient A10 system. NMR spectra were recorded on a Bruker AVANCE spectrometer $(400 \mathrm{MHz}$ for ${ }^{1} \mathrm{H}$ and $100 \mathrm{MHz}$ for ${ }^{13} \mathrm{C}$ ); ${ }^{1} \mathrm{H}$ and ${ }^{13} \mathrm{C}$ spectra were recorded in $\mathrm{CDCl}_{3}, \mathrm{CD}_{3} \mathrm{OD}$, and DMSO- $d_{6}$ at $25^{\circ} \mathrm{C}$. Chemical shifts of ${ }^{13} \mathrm{C}$ are given relative to $\mathrm{CDCl}_{3}, \mathrm{CD}_{3} \mathrm{OD}$, and DMSO- $d_{6}$ as internal standards $(\delta 77.0,849.0, \delta 39.7 \mathrm{ppm})$. Mass spectra were measured on a JEOL JMS-T100GCv MS detector (gas chromatography (GC)-MS) and a JEOL JMS-T100LP MS detector (LC-MS); the base peak is denoted as "bp." GC and IR analyses were performed on a Shimadzu GC-2014 instrument and a Jasco FTIR-410 detector, respectively. ICP-AES spectra were measured on a Shimadzu ICPE-9000 instrument.

\subsection{Materials}

The PS-PEG-supported phenanthroline-palladium complex (PS-PEG-phenanthroline-Pd; 1b) was prepared from a PS-PEG amino-resin (TentaGel $\mathrm{S} \mathrm{NH}_{2}$, average diameter $90 \mu \mathrm{m}, 1 \%$ divinylbenzene cross-linked, loading value of amino residue $0.29 \mathrm{mmol} / \mathrm{g}$; purchased from Rapp Polymer), a polymeric phenanthroline ligand, and $\left(\mathrm{C}_{6} \mathrm{H}_{5} \mathrm{CN}\right)_{2} \mathrm{PdCl}_{2}$ in accordance with previously reported procedures [26]. The loading level of $\mathrm{Pd}$ in polymeric catalyst $1 \mathbf{b}$ was $0.26 \mathrm{mmol} / \mathrm{g}$. 


\subsection{Synthesis of Polymer-Supported Ligand}

First, 5-methyl-1,10-phenanthroline $(99 \mathrm{mg}, 0.51 \mathrm{mmol})$ and $\mathrm{SeO}_{2}(115 \mathrm{mg}, 1.0 \mathrm{mmol})$ were suspended in ortho-dichlorobenzene $(20 \mathrm{~mL})$ and the mixture was heated at reflux for $6 \mathrm{~h}$, and then cooled to room temperature. Filtration was followed by the addition of $5 \mathrm{M}$ aqueous citric acid $(10 \mathrm{~mL})$ to the filtrate. The aqueous phase was collected and washed with dichloromethane and then neutralized by the addition of $10 \mathrm{M} \mathrm{NaOHaq}(15 \mathrm{~mL})$. Dichloromethane was added, and extraction was repeated 5 times with dichloromethane. The corrected organic layers were washed with saturated $\mathrm{NaCl}$ and dried over $\mathrm{MgSO}_{4}$. The solvent was removed by an evaporator and dried in vacuum to give a $76 \%$ yield of 1,10-phenanthroline-5-carbaldehyde. ${ }^{1} \mathrm{H}$ NMR $\left(\mathrm{CDCl}_{3}\right): \delta 10.42(\mathrm{~s}, 1 \mathrm{H}), 9.81(\mathrm{dd}, J=8.1,1.3 \mathrm{~Hz}, 1 \mathrm{H})$, $9.38(\mathrm{dd}, J=3.9,1.3 \mathrm{~Hz}, 1 \mathrm{H}), 9.33(\mathrm{~d}, J=2.7 \mathrm{~Hz}, 1 \mathrm{H}), 8.47(\mathrm{dd}, J=8.0,1.4 \mathrm{~Hz}, 1 \mathrm{H}), 8.41(\mathrm{~s}, 1 \mathrm{H}), 7.83-7.79$ $(\mathrm{m}, 2 \mathrm{H}) ;{ }^{13} \mathrm{C}$ NMR $\left(\mathrm{CDCl}_{3}\right):$ 8192.6, 153.4, 151.1, 148.0, 146.0, 139.7, 137.5, 134.2, 130.4, 126.8, 125.5, 124.4, 123.9; IR (ATR) $\left(\mathrm{cm}^{-1}\right): v 2970,1681,1506$; HR-EI-MS: Calculated for $\mathrm{C}_{13} \mathrm{H}_{9} \mathrm{~N}_{2} \mathrm{O}(\mathrm{M}+)$ 208.0636, found 208.0637.

To a solution of the crude 1,10-phenanthroline-5-carbaldehyde $(81 \mathrm{mg}, 0.39 \mathrm{mmol})$ in THF $(9.0 \mathrm{~mL})$ and $t-\mathrm{BuOH}(9.0 \mathrm{~mL}), 2$-methyl-2-butene $(318 \mathrm{mg}, 4.5 \mathrm{mmol})$ was added. The solution of $\mathrm{NaClO}_{2}$ (105 mg, $1.2 \mathrm{mmol})$ and $\mathrm{NaH}_{2} \mathrm{PO}_{4} \cdot 2 \mathrm{H}_{2} \mathrm{O}(182 \mathrm{mg}, 1.2 \mathrm{mmol})$ in $3.0 \mathrm{~mL}$ water was added to the reaction mixture and the mixture was stirred vigorously at $25^{\circ} \mathrm{C}$ for $24 \mathrm{~h}$, after which a white suspension was obtained. To a reaction mixture, $43 \mathrm{~mL}$ of $0.70 \mathrm{M} \mathrm{NaOH}$ was added, and the combined reaction mixture was washed with $\mathrm{CH}_{2} \mathrm{Cl}_{2}$ to remove residual starting materials. The water layers were neutralized with $10 \mathrm{~mL}$ of $0.5 \mathrm{M}$ critic acid to generate a white precipitate via crystallization at $0{ }^{\circ} \mathrm{C}$. The precipitate was filtered and washed with water. The precipitate was further purified by heating under reflux for $1 \mathrm{~h}$ in $\mathrm{MeOH}$. The 5-carboxy-1,10-phenanthroline (13.7 mg, 16\% yield) was collected by filtration. ${ }^{1} \mathrm{H}-\mathrm{NMR}\left(\mathrm{CD}_{3} \mathrm{OD}\right): 89.52(\mathrm{dd}, J=8.5,1.6 \mathrm{~Hz}, 1 \mathrm{H}), 9.17(\mathrm{dd}, J=4.4,1.6 \mathrm{~Hz}, 1 \mathrm{H}), 9.11(\mathrm{dd}, J=4.2,1.6 \mathrm{~Hz}$, $1 \mathrm{H}), 8.72-8.69(\mathrm{~m}, 1 \mathrm{H}), 8.59(\mathrm{dd}, J=8.0,1.6 \mathrm{~Hz}, 1 \mathrm{H}), 7.85-7.82(\mathrm{~m}, 2 \mathrm{H}) ;{ }^{13} \mathrm{C}-\mathrm{NMR}$ (DMSO- $\left.d_{6}\right): 8.168 .0$, 152.5, 150.4, 147.2, 146.0, 138.1, 134.8, 132.1, 127.1, 126.8, 126.6, 124.3, 124.0; IR (ATR) ( $\mathrm{cm}^{-1}$ ): $v 3669$ (br), 2997, 1683, 1508; HR-ESI-MS: Calculated for $\mathrm{C}_{13} \mathrm{H}_{9} \mathrm{~N}_{2} \mathrm{O}_{2}(\mathrm{M}+\mathrm{H}) 225.0664$, found 225.0665 .

\subsection{Preparation of PS-PEG Resin-Supported Phenanthroline-Palladium Complex $\mathbf{1 b}$}

A Merrifield vessel was charged with PS-PEG- $\mathrm{NH}_{2}(348 \mathrm{mg}, 0.10 \mathrm{mmol})$, phenanthroline-COOH ligand (35.0 mg, $0.16 \mathrm{mmol})$, 1-ethyl-3-(3-dimethylaminopropyl) carbodiimide (39.4 mg, $0.21 \mathrm{mmol}$ ), 1-hydroxybenzotriazole $(27.4 \mathrm{mg}, 0.2 \mathrm{mmol})$, and DMSO $(6 \mathrm{~mL})$. The reaction mixture was shaken at $25^{\circ} \mathrm{C}$ for $17 \mathrm{~h}$. The consumption of the primary amino residue of the resin was monitored by the Kaiser negative test. The reaction mixture was filtered, and the resin was washed with DMSO and $\mathrm{CH}_{2} \mathrm{Cl}_{2}$. The resin was dried under reduced pressure to provide the polymer-supported phenanthroline.

Another Merrifield vessel was charged with the obtained resin-supported phenanthroline ligand $(0.10 \mathrm{mmol})$ and toluene $(6 \mathrm{~mL})$. To a suspension, $\left(\mathrm{C}_{6} \mathrm{H}_{5} \mathrm{CN}\right)_{2} \mathrm{PdCl}_{2}(45.9 \mathrm{mg}, 0.12 \mathrm{mmol})$ was added, and the mixture was shaken $(\mathrm{CM}-1000)$ at $25^{\circ} \mathrm{C}$ for $2 \mathrm{~h}$. The mixture was filtered, and the resin was washed with toluene and $\mathrm{CH}_{2} \mathrm{Cl}_{2}$. The resulting resin was dried under reduced pressure to provide the polymer-supported palladium complex $\mathbf{1 b}$ (loading value of Pd: $0.26 \mathrm{mmol} / \mathrm{g}$ ). IR (ATR) $\left(\mathrm{cm}^{-1}\right)$; 1; v 2868, 1683, 1102, 700 .

\subsection{Palladium-Catalyzed Friedel-Crafts-Type Alkylation of Indoles with Allyl Esters}

The reaction conditions and results are shown in Table 1. A typical procedure is given for the reaction with indole $\mathbf{2} \mathbf{a}$ and 1,3-diphenyl-2-propenyl acetate $\mathbf{3 a}$ in water in entry 1 . To a solution of catalyst 1 ( $38.0 \mathrm{mg}, 0.01 \mathrm{mmol}), 1,3$-diphenyl-2-propenyl acetate $(75 \mathrm{mg}, 0.30 \mathrm{mmol})$, and indole $2 \mathrm{a}$ (23.4 mg, $0.2 \mathrm{mmol})$ in $\mathrm{H}_{2} \mathrm{O}(1.5 \mathrm{~mL})$, triethylamine $(60.7 \mathrm{mg}, 0.60 \mathrm{mmol})$ was added, and the mixture was stirred at $40{ }^{\circ} \mathrm{C}$ for $24 \mathrm{~h}$. The reaction mixture was filtered and the recovered resin beads were rinsed three times with AcOEt. The combined filtrate was extracted with AcOEt. The combined extracts were washed with aqueous sodium chloride and dried over anhydrous magnesium sulfate. 
The solvent was evaporated and the residue was chromatographed on silica gel (hexane/ethyl acetate $=$ 95/5) to give a mixture of the corresponding substituted products and indole. The indole was removed from a mixture of products and indole by distillation to give 3-(1,3-diphenyl-2-propenyl)-1H-indole in $88 \%$ yield ( $54.5 \mathrm{mg}, 0.18 \mathrm{mmol}$ ). (To remove the other indoles $\mathbf{2} \mathbf{b}-\mathbf{h}$, the mixture of products and indoles was separated by recycle HPLC (JAI LC-9201)).

The compounds and CAS registry numbers are as follows: 3-(1,3-diphenyl-2-propenyl)- $1 H$-indole (4a), 3-(1,3-diphenyl-2-propenyl)-2-methyl-1H-indole (4b), 3-(1,3-diphenyl-2-propenyl)-2-phenyl-1H-indole (4c), 3-(1,3-diphenyl-2-propenyl)-5-methyl-1H-indole (4d), 3-(1,3-diphenyl-2-propenyl)-7-methyl-1H-indole (4e), 3-(1,3-diphenyl-2-propenyl)-5-bromo- $1 \mathrm{H}$-indole (4f), 3-(1,3-diphenyl-2-propenyl)-5- chloro- $\mathrm{H}$-indole (4g), 3-(1,3-diphenyl-2-propenyl)-5-methoxy- $1 H$-indole (4h), 3-(1,3-diphenyl-2-propenyl)-5-benzyloxy$1 H$-indol (4i); and 1265313-04-9, 956480-06-6, 769929-40-0, 1264750-28-8, 1264750-28-8, 769929-44-4, 1264750-26-6, 957777-42-3, 769929-42-2, respectively.

Spectral and analytical data for the 4 are shown below:

(R)-3-(1,3-diphenyl-2-propenyl)-1H-indole (4a): ${ }^{1} \mathrm{H}$ NMR $\left(\mathrm{CDCl}_{3}\right)$ : $\delta 8.01$ (br, $\left.1 \mathrm{H}\right), 7.27-7.44$ (m, 14 H), 7.16-7.24 (m, $1 \mathrm{H}), 6.73(\mathrm{dd}, J=7.4,15.8 \mathrm{~Hz}, 1 \mathrm{H}), 6.45(\mathrm{~d}, J=15.1 \mathrm{~Hz}, 1 \mathrm{H}), 5.13(\mathrm{~d}, J=7.3 \mathrm{~Hz}, 1 \mathrm{H})$. ${ }^{13} \mathrm{C} \mathrm{NMR}\left(\mathrm{CDCl}_{3}\right)$ : $\delta 143.3,137.5,136.6,132.5,130.5,128.5(2 \mathrm{C}), 128.4(2 \mathrm{C}), 127.1(2 \mathrm{C}), 126.7(2 \mathrm{C}), 126.4$ (2C), 126.3, 122.6, 122.1, 119.9, 119.4, 118.7, 111.1, 46.2. MS (EI): $\mathrm{m} / \mathrm{z}$ (rel\%) 309 (bp, $\mathrm{M}^{+}$), 232 (36), 130 (30), 130 (29), 77 (16). IR (ATR): ( $\left(\mathrm{cm}^{-1}\right) v 3419$ (br), 3056, 3024. EI-HRMS: Calcd for $\mathrm{C}_{23} \mathrm{H}_{19} \mathrm{~N}$ 309.1517, found 309.1516 .

3-(1,3-diphenyl-2-propenyl)-2-methyl-1H-indole (4b): ${ }^{1} \mathrm{H}$ NMR $\left(\mathrm{CDCl}_{3}\right): \delta 7.62(\mathrm{br}, 1 \mathrm{H}), 7.36-7.31$ $(\mathrm{m}, 5 \mathrm{H}), 7.26-7.20(\mathrm{~m}, 4 \mathrm{H}), 7.18-7.14(\mathrm{~m}, 3 \mathrm{H}), 7.05(\mathrm{td}, J=7.1,1.1 \mathrm{~Hz}, 1 \mathrm{H}), 6.95(\mathrm{td}, J=7.1,1.1 \mathrm{~Hz}$, $1 \mathrm{H}), 6.81(\mathrm{dd} J=15.8,7.2 \mathrm{~Hz}, 1 \mathrm{H}), 6.39(\mathrm{~d}, J=15.8 \mathrm{~Hz}, 1 \mathrm{H}), 5.11(\mathrm{~d}, J=7.2 \mathrm{~Hz}, 1 \mathrm{H}), 2.27(\mathrm{~s}, 3 \mathrm{H})$. ${ }^{13} \mathrm{C}$ NMR $\left(\mathrm{CDCl}_{3}\right): 8143.4,137.4,135.2,132.1,131.5,130.5,128.4(2 \mathrm{C}), 128.2(2 \mathrm{C}), 127.8(2 \mathrm{C}), 127.0(2 \mathrm{C})$, 126.2 (2C), 126.0, 120.8, 119.3, 119.1, 112.7, 110.2, 45.0, 12.2. MS (EI): $\mathrm{m} / \mathrm{z}(\mathrm{rel} \%) 323$ (bp, $\left.\mathrm{M}^{+}\right), 308$ (67), 246 (38), 218 (36), 144 (42), 77 (14). IR (ATR): $\left(\mathrm{cm}^{-1}\right) v 3379$ (br), 2982. EI-HRMS: Calcd for $\mathrm{C}_{24} \mathrm{H}_{21} \mathrm{~N}$ 323.1674, found 323.1682 .

3-(1,3-diphenyl-2-propenyl)-2-phenyl-1H-indole (4c): ${ }^{1} \mathrm{H}$ NMR $\left(\mathrm{CDCl}_{3}\right): 88.05$ (br, $\left.1 \mathrm{H}\right), 7.53-7.51$ (m, $2 \mathrm{H}), 7.45-7.41(\mathrm{~m}, 3 \mathrm{H}), 7.38-7.31(\mathrm{~m}, 6 \mathrm{H}), 7.27-7.23(\mathrm{~m}, 4 \mathrm{H}), 7.20-7.14(\mathrm{~m}, 3 \mathrm{H}), 6.99(\mathrm{td}, J=8.0$, $1.0 \mathrm{~Hz}, 1 \mathrm{H}), 6.88(\mathrm{dd}, J=15.8,7.2 \mathrm{~Hz}, 1 \mathrm{H}), 6.39(\mathrm{dd}, J=15.8,1.0 \mathrm{~Hz}, 1 \mathrm{H}), 5.27(\mathrm{~d}, J=7.2 \mathrm{~Hz}, 1 \mathrm{H}) .{ }^{13} \mathrm{C}$ NMR $\left(\mathrm{CDCl}_{3}\right)$ : $\delta 143.4,137.4,136.2,135.5,132.9,132.2,131.0,128.7$ (2C), 128.5 (2C), $128.4(2 \mathrm{C}), 128.2$ (2C), 128.2, 128.0, 127.9, 127.0, 126.2 (2C), 126.0, 122.0, 121.2, 119.6, 113.8, 110.9, 45.1. MS (EI): $\mathrm{m} / \mathrm{z}(\mathrm{rel} \%)$ 385 (bp, M ${ }^{+}$), 294 (89), 206 (41), 91 (13). IR (ATR): $\left(\mathrm{cm}^{-1}\right) v 3408$ (br), 3056, 3023. EI-HRMS: Calcd for $\mathrm{C}_{29} \mathrm{H}_{23} \mathrm{~N} 385.1830$, found 385.1835.

3-(1,3-diphenyl-2-propenyl)-5-methyl-1H-indole (4d): ${ }^{1} \mathrm{H}$ NMR $\left(\mathrm{CDCl}_{3}\right)$ : $\delta 7.74$ (br, $\left.1 \mathrm{H}\right), 7.35-7.16$ $(\mathrm{m}, 12 \mathrm{H}), 6.97(\mathrm{dd}, J=8.2,1.4 \mathrm{~Hz}, 1 \mathrm{H}), 6.77(\mathrm{dd}, J=2.3,0.6 \mathrm{~Hz}, 1 \mathrm{H}), 6.69(\mathrm{dd}, J=15.8,7.2 \mathrm{~Hz}, 1 \mathrm{H})$, $6.39(\mathrm{dd}, J=16.4 \mathrm{~Hz}, 1 \mathrm{H}), 5.07(\mathrm{~d}, J=7.2 \mathrm{~Hz}, 1 \mathrm{H}), 2.35(\mathrm{~s}, 3 \mathrm{H}) .{ }^{13} \mathrm{C} \mathrm{NMR}\left(\mathrm{CDCl}_{3}\right): 8143.4,137.5,134.9$, 132.6, 130.4, 128.5 (2C), 128.4 (2C), 128.4, 128.3 (2C), 127.0, 127.0, 126.2, 126.2 (2C), 123.7, 122.7, 119.3, 118.0, 110.7, 46.0, 21.5. MS (EI): $\mathrm{m} / \mathrm{z}$ (rel\%) 323 (bp, $\mathrm{M}^{+}$), 246 (42), 220 (28), 191 (20), 144 (14), 91 (8). IR (ATR): $\left(\mathrm{cm}^{-1}\right) \cup 3404$ (br), 2982. EI-HRMS: Calcd for $\mathrm{C}_{24} \mathrm{H}_{21} \mathrm{~N}$ 323.1674, found 323.1679.

3-(1,3-diphenyl-2-propenyl)-7-methyl-1H-indole (4e): ${ }^{1} \mathrm{H}$ NMR $\left(\mathrm{CDCl}_{3}\right): \delta 7.69$ (br, $\left.1 \mathrm{H}\right), 7.33-7.10$ $(\mathrm{m}, 11 \mathrm{H}), 6.95-6.91(\mathrm{~m}, 2 \mathrm{H}), 6.76(\mathrm{dd}, J=2.4,0.8 \mathrm{~Hz}, 1 \mathrm{H}), 6.69(\mathrm{dd}, J=15.8,7.3 \mathrm{~Hz}, 1 \mathrm{H}), 6.40(\mathrm{dd}, J=$ 15.8, $0.5 \mathrm{~Hz}, 1 \mathrm{H}), 5.07(\mathrm{~d}, J=7.3 \mathrm{~Hz}, 1 \mathrm{H}), 2.37(\mathrm{~s}, 3 \mathrm{H}) .{ }^{13} \mathrm{C} \mathrm{NMR}\left(\mathrm{CDCl}_{3}\right): 8143.4,137.4,136.1,132.5$, 130.4, 128.4 (2C), 128.4 (2C), 128.3 (2C), 127.0, 126.3, 126.3, 126.2 (2C), 122.5, 122.2, 120.1, 119.6, 119.0, 117.5, 46.2, 16.4. MS (EI): $\mathrm{m} / \mathrm{z}$ (rel\%) 323 (bp, M+ ), 246 (38), 220 (28), 191 (20), 144 (22), 91 (7). IR (ATR): $\left(\mathrm{cm}^{-1}\right) v 3429$ (br), 3024. EI-HRMS: Calcd for $\mathrm{C}_{24} \mathrm{H}_{21} \mathrm{~N}$ 323.1674, found 323.1670.

3-(1,3-diphenyl-2-propenyl)-5-bromo-1H-indole (4f): ${ }^{1} \mathrm{H}$ NMR $\left(\mathrm{CDCl}_{3}\right): \delta 7.84(\mathrm{br}, 1 \mathrm{H}), 7.52(\mathrm{~d}, J=$ $1.8 \mathrm{~Hz}, 1 \mathrm{H}), 7.33-7.16(\mathrm{~m}, 11 \mathrm{H}), 7.10(\mathrm{~d}, J=8.8 \mathrm{~Hz}, 1 \mathrm{H}), 6.79(\mathrm{~d}, J=2.4 \mathrm{~Hz}, 1 \mathrm{H}), 6.64(\mathrm{dd}, J=15.8$, $7.3 \mathrm{~Hz}, 1 \mathrm{H}), 6.37(\mathrm{~d}, J=15.8 \mathrm{~Hz}, 1 \mathrm{H}), 5.00(\mathrm{~d}, J=7.2 \mathrm{~Hz}, 1 \mathrm{H}) .{ }^{13} \mathrm{C} \mathrm{NMR}\left(\mathrm{CDCl}_{3}\right): \delta 142.8,137.2,135.1$, 
132.0, 130.0, $128.4(2 \mathrm{C}), 128.4,128.4(2 \mathrm{C}), 128.3$ (2C), 127.2, 126.5, 126.2 (2C), 124.9, 123.7, 122.1, 118.2, 112.6, 112.5, 45.8. MS (EI): $\mathrm{m} / \mathrm{z}$ (rel\%) 389 (42), $387\left(\mathrm{M}^{+}, 47\right), 231$ (25), 233 (25), 130 (45), 68 (bp). IR (ATR): $\left(\mathrm{cm}^{-1}\right)$ v 3364 (br), 2982. EI-HRMS: Calcd for $\mathrm{C}_{23} \mathrm{H}_{18}{ }^{79} \mathrm{BrN}$ 387.0622, found 387.0638.

3-(1,3-diphenyl-2-propenyl)-5-chloro-1 $H$-indole $(4 \mathrm{~g}):{ }^{1} \mathrm{H}$ NMR $\left(\mathrm{CDCl}_{3}\right): \delta 7.84(\mathrm{br}, 1 \mathrm{H}), 7.37-7.14(\mathrm{~m}$, $12 \mathrm{H}), 7.07(\mathrm{dd}, J=8.6,1.9 \mathrm{~Hz}, 1 \mathrm{H}), 6.81(\mathrm{dd}, J=2.2,0.5 \mathrm{~Hz}, 1 \mathrm{H}), 6.64(\mathrm{dd}, J=15.8,7.3 \mathrm{~Hz}, 1 \mathrm{H}), 6.38$ $(\mathrm{d}, J=15.8 \mathrm{~Hz}, 1 \mathrm{H}), 5.01(\mathrm{~d}, J=7.3 \mathrm{~Hz}, 1 \mathrm{H}) .{ }^{13} \mathrm{C} \mathrm{NMR}\left(\mathrm{CDCl}_{3}\right): \delta 142.8,137.2,134.9,132.0,130.7,128.4$ (2C), 128.4 (2C), 128.3 (2C), 127.8, 127.2, 126.5, 126.2 (2C), 125.0, 123.9, 122.3, 119.1, 118.3, 112.1, 45.8. MS (EI): $\mathrm{m} / z$ (rel\%) 343 (bp, M+), 266 (35), 240 (29), 191 (27), 115 (26). IR (ATR): (cm ${ }^{-1}$ ) $v 3853$ (br), 3025. EI-HRMS: Calcd for $\mathrm{C}_{23} \mathrm{H}_{18}{ }^{35} \mathrm{ClN} 343.1127$, found 343.1131.

3-(1,3-diphenyl-2-propenyl)-5-methoxy-1H-indole (4h): ${ }^{1} \mathrm{H}$ NMR $\left(\mathrm{CDCl}_{3}\right): 87.75$ (br, $\left.1 \mathrm{H}\right), 7.34-7.11$ $(\mathrm{m}, 12 \mathrm{H}), 6.84-6.65(\mathrm{~m}, 4 \mathrm{H}), 6.41(\mathrm{~d}, J=15.8,1 \mathrm{H}), 5.03(\mathrm{~d}, J=7.2 \mathrm{~Hz}, 1 \mathrm{H}), 3.67(\mathrm{~s}, 3 \mathrm{H}) .{ }^{13} \mathrm{C}$ NMR $\left(\mathrm{CDCl}_{3}\right):$ : $153.7,143.2,137.4,132.4,131.7,130.4,128.4(2 \mathrm{C}), 128.3(3 \mathrm{C}), 127.1(2 \mathrm{C}), 126.3(2 \mathrm{C}), 126.2(2 \mathrm{C})$, 123.4, 118.1, 112.0, 111.7, 101.8, 55.7, 46.1. MS (EI): m/z (rel\%) 339 (bp, $\mathrm{M}^{+}$), 262 (38), 236 (25), 115 (12). IR (ATR): $\left(\mathrm{cm}^{-1}\right) v 3420$ (br), 3024. EI-HRMS: Calcd for $\mathrm{C}_{24} \mathrm{H}_{21} \mathrm{NO} 339.1623$, found 339.1624.

3-(1,3-diphenyl-2-propenyl)-5-benzyloxy-1H-indole (4i): ${ }^{1} \mathrm{H}$ NMR $\left(\mathrm{CDCl}_{3}\right): 87.66$ (br, $\left.1 \mathrm{H}\right), 7.34-7.13$ $(\mathrm{m}, 15 \mathrm{H}), 7.06(\mathrm{~d}, J=8.8 \mathrm{~Hz}, 1 \mathrm{H}), 6.92(\mathrm{~d}, J=2.3 \mathrm{~Hz}, 1 \mathrm{H}), 6.85(\mathrm{dd}, J=8.7,2.4 \mathrm{~Hz}, 1 \mathrm{H}), 6.99(\mathrm{~d}$, $J=2.0 \mathrm{~Hz}, 1 \mathrm{H}), 6.64(\mathrm{dd}, J=15.8,7.3 \mathrm{~Hz}, 1 \mathrm{H}), 6.38(\mathrm{~d}, J=15.8 \mathrm{~Hz}, 1 \mathrm{H}), 4.99(\mathrm{~d}, J=7.3 \mathrm{~Hz}, 1 \mathrm{H})$, 4.90 (s, $2 \mathrm{H}) .{ }^{13} \mathrm{C}$ NMR $\left(\mathrm{CDCl}_{3}\right): 8152.8,143.2,137.5,137.4,132.4,131.8,130.4,128.4(2 \mathrm{C}), 128.3(2 \mathrm{C})$, 128.3, 127.6 (2C), 127.5 (2C), 127.0 (2C), 127.0 (2C), 126.3, 126.2 (2C), 123.4, 118.0, 112.8, 111.7, 103.3, 70.8, 46.1. MS (ESI): $\mathrm{m} / z$ (rel\%) 438 (bp, M+Na). IR (ATR): $\left(\mathrm{cm}^{-1}\right) v 3426$ (br), 3025. ESI-HRMS: Calcd for $\mathrm{C}_{30} \mathrm{H}_{25} \mathrm{NONa} 438.1833$, found 438.1836 .

\section{Conclusions}

In summary, we have developed a practical protocol for the Friedel-Crafts-type alkylation of indoles with allylic ester using the PS-PEG resin-supported phenanthroline-palladium complex to give the 3-allyl- $1 H$-indoles with up to $91 \%$ yield. This polymeric catalyst was also found to promote the C3-alkylation reaction to give a thermodynamic alkylation product with high selectivity. This catalyst was recovered and reused several times without any loss of activity.

Author Contributions: T.S. designed and performed the research and experiments and did the data analysis, manuscript writing, and revision. Y.O. performed the experiments and analyzed the data. K.O. advised us for this research. All authors have read and agreed to the published version of the manuscript.

Acknowledgments: We are grateful for financial support from the Steel Foundation for Environmental Protection Technology. We thank Hiromu Sueyoshi for assistance with some of the lab works.

Conflicts of Interest: The authors declare no conflict of interest.

\section{References}

1. Calloway, N.O. The Friedel-Crafts Synthesis. Chem. Rev. 1935, 17, 327-392. [CrossRef]

2. Friedel, C.; Crafts, J.M. A new general synthetical method of producing hydrocarbons. Hebd. Seances Acad. Sci. $\mathbf{1 8 7 7 , 8 4 , 1 3 9 2 - 1 3 9 5 . ~}$

3. Ohal, G.A.; Krishnamurti, R.; Prakash, G.K.S. Comprehensive Organic Synthesis; Trost, B.M., Prakash, G.K.S., Eds.; Pergamon: Oxford, UK, 1991; Volume 3, p. 293.

4. Bandini, M.; Melloni, A.; Umani-Ronchi, A. New catalytic approaches in the stereoselectitive Friedel-Crafts alkylation reaction. Angew. Chem. Int. Ed. 2004, 43, 550-556. [CrossRef] [PubMed]

5. Trost, B.M.; Van Vranken, D.L. Asynmetric transition metal-catalyzed allylic alkylations. Chem. Rev. 1996, 96, 395-422. [CrossRef] [PubMed]

6. Trost, B.M. On Inventing Reaction for Atom Economy. Acc. Chem. Res. 2002, 35, 695-705. [CrossRef] [PubMed]

7. Trost, B.M.; Crawly, M.L. Asymmetric Transition-metal-catalyzed allylic alkylations: Applications in total synthesis. Chem. Rev. 2003, 103, 2921-2944. [CrossRef] 
8. Malkov, A.V.; Davis, S.L.; Baxendale, I.R.; Mitchell, W.L.; Kočovský, P. Molybdenum(II)-catalyzed allylation of electron-rich aromatics and heteroaromatics. J. Org. Chem. 1999, 64, 2751-2764. [CrossRef]

9. Bandini, M.; Melloni, A.; Umani-Ronchi, A. New versatile Pd-catalyzed alkylation of indoles via nucleophilic allylic substitution: Controlling the regioselectivity. Org. Lett. 2004, 6, 3199-3202. [CrossRef]

10. Bandini, M.; Melloni, A.; Piccinelli, F.; Sinisi, R.; Tommasi, S.; Umani-Ronchi, A. Highly enantioselective synthesis of tetrahydro-ß-carbolines and tetrahydro- $\gamma$-carbolines via Pd-catalyzed intramolecular allylic alkylation. J. Am. Chem. Soc. 2006, 128, 1424-1425. [CrossRef]

11. Ma, S.; Yu, S.; Peng, Z.; Guo, H. Palladium-catalyzed functionalization of indoles with 2-acetoxymethylsubstitued electron-deficient alkenes. J. Org. Chem. 2006, 71, 9865-9868. [CrossRef]

12. Cheung, H.Y.; Yu, W.-Y.; Lam, F.L.; Au-Yeung, T.T.-L.; Zhou, Z.; Chan, T.H.; Chan, A.S.C. Enantioselective Pd-catalyzed allylic alkylation of indoles by a new class of chiral ferrocenyl P/S ligands. Org. Lett. 2007, 9, 4295-4298. [CrossRef] [PubMed]

13. Kimura, M.; Futamata, M.; Mukai, R.; Tamaru, Y. Palladium-catalyzed enantioselective C-3 allylation of 3-substituted-1H-indoles using trialkylboranes. J. Am. Chem. Soc. 2006, 128, 6314-6315.

14. Cao, Z.; Liu, Y.; Liu, Z.; Feng, X.; Zhuang, M.; Du, H. Pd-catalyzed asymmetric allylic alkylation of indoles and pyrroles by chiral alkene-phosphine ligands. Org. Lett. 2011, 13, 2164-2167. [CrossRef] [PubMed]

15. Trillo, P.; Baeza, A.; Nájera, C. Fluorinated alcohols as promoters for the metal-free direct substitution reaction of allylic alcohols with nitrogenated, silylated, and carbon nucleophiles. J. Org. Chem. 2012, 77, 7344-7354. [CrossRef]

16. Peng, B.J.; Hsueh, W.T.; Fülöp, F.; Yang, S.C. Platinum-catalyzed selective N-allylation of 2,3-disubstitued indoles with allylic acetates in water. New. J. Chem. 2019, 43, 58-62. [CrossRef]

17. Peng, B.J.; Huang, Y.T.; Fülöp, F.; Lin, I.L.; Yang, S.C. Palladium-catalyzed selective N-allylation of indoles assiseted by PEG-water system. New J. Chem. 2019, 29, 11549-11553. [CrossRef]

18. Suzuka, T.; Okada, Y.; Ooshiro, K.; Uozumi, Y. Copper-free Sonogashira coupling in water with an amphiphilic resin-supported palldium complex. Tetrahedron 2010, 66, 1064-1069. [CrossRef]

19. Suzuka, T.; Kawahara, Y.; Ooshiro, K.; Nagamine, T.; Ogihara, K.; Higa, M. Reusable polymer-Ssupported 2,2'-biarylpyridine-copper complexes for Huisgen [3+2] cycloaddition in water. Heterocycles 2012, 3, 615-626. [CrossRef]

20. Suzuka, T.; Nagamine, T.; Ogihara, K.; Higa, M. Suzuki-Miyaura Cross coupling reaction in water with polymer-supported terpyridine palladium complex under aerobic conditions. Catal. Lett. 2010, 139, 85-89. [CrossRef]

21. Suzuka, T.; Nagamine, T.; Ogihara, K.; Higa, M. Mizoroki-Heck Reaction in water with polymer-supported terpyridine palladium complex under aerobic conditions. Trans. Mater. Res. Soc. Jpn. 2010, 35, 889-892. [CrossRef]

22. Suzuka, T.; Kimura, K.; Nagamine, T. Reusable polymer-supported terpyridine palladium complex for the Suzuki-Miyaura, Mizoroki-Heck, Sonogashira, Tsuji-Trost coupling reaction in Water. Polymers 2011, 3, 621-639. [CrossRef]

23. Suzuka, T.; Adachi, M.; Yang, Z.-S.; Ogihara, K.; Higa, M. Suzuki-Miyaura cross coupling reaction in water with polymer-supported terpyridine palladium complex and application for the synthesis of 2,6-disubstitued pyrimidines. Trans. Mater. Res. Soc. Jpn. 2013, 38, 119-122. [CrossRef]

24. Suzuka, T.; Adachi, M.; Nakamoto, Y.; Ogihara, K. Use of polymer-supported terpyridine-Palladium complex for Mizoroki-Heck reaction in water under aerobic conditions. Trans. Mater. Res. Soc. Jpn. 2015, 40, 77-80. [CrossRef]

25. Suzuka, T.; Adachi, M.; Ogihara, K. Sonogashira coupling reaction in water with polymer-supported terpyridine-palladium complex under aerobic conditions. Trans. Mater. Res. Soc. Jpn. 2015, 40, 103-106. [CrossRef]

26. Suzuka, T.; Sueyoshi, H.; Maehara, S.; Ogasawara, H. Reactivity of aryl halides for transfer reduction in (sea) water using polymer-supported terpyridine palladium complex. Molecules 2015, 20, 9906-9914. [CrossRef]

27. Suzuka, T.; Sueyoshi, H.; Ogiahra, K. Polymer-supported terpyridine-palladium complex for the aminocarbonylation in water of aryl iodides using methoxylamine hydrochloride as an ammonia equivalent. Trans. Mater. Res. Soc. Jpn. 2016, 41, 225-228. [CrossRef] 
28. Suzuka, T.; Sueyoshi, H.; Ogihara, K. Recyclable polymer supported terpyridine palladium complex for the tandem aminocarbonylation of aryl iodides using $\mathrm{NaN}_{3}$ as an ammonia equivalent. Catalysts 2017, 7, 107-115. [CrossRef]

29. Li, C.-J.; Chan, T.-H. Organic Reactions in Aqueous Media; Wiley-VCH: New York, NY, USA, 1997.

30. Grieco, P.A. Organic Synthesis in Water; Kluwer Academic Publishers: Dordrecht, The Netherlands, 1997.

31. Herrmann, W.A.; Kohlpaintner, C.W. Water-soluble ligands, metal complexes, and catalysts: Synergism of homogeneous and heterogeneous catalyst. Angew. Chem. Int. Ed. Engl. 1993, 32, 1524-1544. [CrossRef]

32. Lindström, U.M. Stereoselective organic reaction in water. Chem. Rev. 2002, 102, 2751-2772. [CrossRef]

33. Bailey, D.C.; Langer, S.H. Immobilized transition-metal carbonyls and related catalysts. Chem. Rev. 1981, 81, 109-148. [CrossRef]

34. Dörwald, F.Z. Organic Synthesis on Solid Phase; Wiley-VCH: Weinheim, Germany, 2000.

35. Leadbeater, N.E.; Marco, M. Preparation of polymer-supported ligands and metal complexes for use in catalysis. Chem. Rev. 2002, 102, 3217-3274. [CrossRef] [PubMed]

36. McNamara, C.A.; Dixon, M.J.; Bradley, M. Recoverable catalysts and reagents using recyclable polystyrene-based supports. Chem. Rev. 2002, 102, 3275-3300. [CrossRef] [PubMed]

(C) 2020 by the authors. Licensee MDPI, Basel, Switzerland. This article is an open access article distributed under the terms and conditions of the Creative Commons Attribution (CC BY) license (http://creativecommons.org/licenses/by/4.0/). 